Mund Kiefer GesıchtsChur (1997) 1:127-129 (C) Sprunger-Verlag 1997

\section{Professor Dr. Dr. Gerhard Grimm (5.1.1926-10.12.1996)}

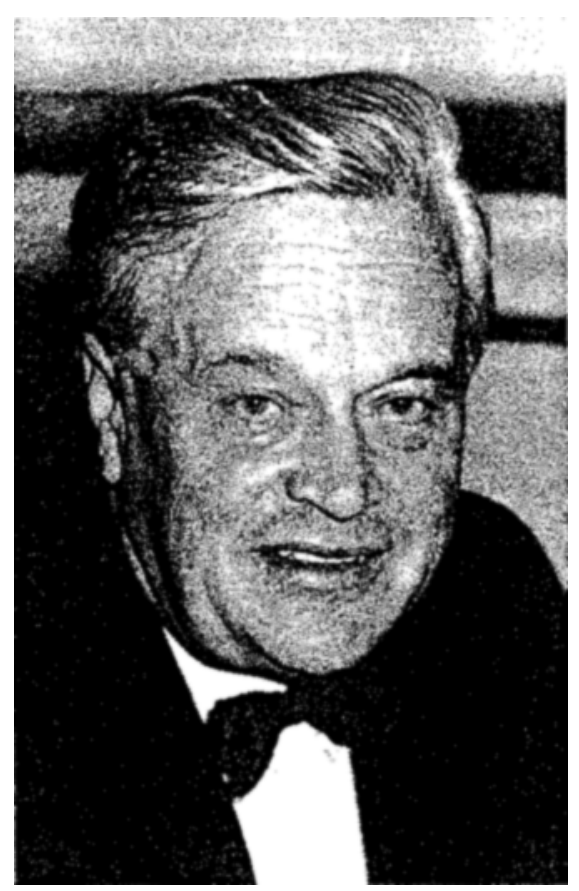

In der unmittelbaren Vorweihnachtszeit 1996 erreichte uns die Nachricht vom Ableben Gerhard Grimms, dem emeritierten Lehrstuhlinhaber für Mund-, Kiefer- und Gesichtschirurgie an der Martin-Luther-Universität Halle-Wittenberg. Trotz eines langen Leidenswegs war es ein unerwarteter Abschied, der Anlaß würdigen Gedenkens ist.

Geboren am 5. Januar 1926 in Oelsnitz, Erzgebirge, legte er 1944 in Stollberg das Abitur $a b$ und konnte von 1947-1953 Medizin und Zahnheilkunde in Rostock studieren. Es folgten die Promotionen zum Dr. med. und Dr. med. dent. Nach einer fundierten 2 jährigen chirurgischen und internistischen Ausbildung am Bergbaukrankenhaus Stollberg konnte er 1955 mit der Weiterbildung zum Facharzt für Zahn-. Mund- und Kieferkrankheiten bei Reichenbach beginnen, die durch eine halbjährige Tätigkeit an der Hals-NasenOhren-Klinik in Halle und einer soliden Ausbildung in moderner Narkosetätigkeit in Berlin-Friedrichshain Ergänzungen erfahren sollte. Nach Facharztanerkennung und Ernennung zum Oberarzt 1958 war er bereits klinisch soweit gefestigt, daß er nach der Zwangsemeritierung von Reichenbach die Klinik leiten und entwickeln konnte. Seine Habilitation, 1968 erfolgreich abgeschlossen, ist das Spiegelbild umfangreicher Erfahrungen und bemerkenswerter Fähigkeiten auf dem Gebiet der experimentellen Forschung. 1974 erfolgte die Berufung zum ordentlichen Professor mit Lehrstuhl.

Seiner Feder entstammen 115 Publikationen. davon 43 in Hand- und Lehrbüchern. Das Studium der Beiträge offenbart nicht nur den Erfahrungsreichtum eines begabten Operateurs. sondern läßt zugleich Meisterwerke stilistischer Gediegenheit erkennen. Von besonderer Bedeutung sind seine Monographie über radiogene Knochenschädigung. der Beitrag ,Plastisch-chirurgische Versorgung ron Strahlenfolgen "im Handbuch der Plastischen Chirurgie von Gohrbandt, Gabka, Berndorfer sowie seine umfangreichen Beitrige in der „Tratmatologie des Gesichtsschädels" unter der Herausgabe von Reichen- bach. Eine bemerkenswerte Leistung fur ein damals junges Fachgebiet war die ,Kinderzahnheilkunde im Vorschulalter". Die ,Zahn-. Mund-und Kieferheilkunde", für deren Editıon er gemeinsam mit Schwenzer verantwortlich zeichnete, gehört inzwischen zu den Standardlehrbuchern in den deutschsprachigen Ländern.

Professor Grimm führte eine Vielzahl von Kollegen zur Promotion. Allein 8 seiner Mitarbeiter sind habilitiert. Verdiente Ehrungen blieben nicht aus. 1970 wurde er zum Mitglied der Deutschen Akademie für Naturforscher LEOPOLDINA gewählt. Nahezu ein Jahrzehnt gehörte er deren Senat an. Seit 1985 war er Ehrenmitglied der Ungarischen Gesellschaft für Stomatologie. Diese Anerkennung wurde ihm auch von der Deutschen Gesellschaft für Mund-, Kiefer- und Gesichtschirurgie zuteil.

Seine Persönlichkeitsstruktur resultierte aus einer weitgefächerten Bildung und vielseitigen Begabung, die nicht auf die Medizin beschränkt waren. Sie umfaßte insbesondere die $\mathrm{Mu}$ sik - seine Leistungen als Pianist verdienen, hervorgehoben $\mathrm{zu}$ werden -, die Kunst zu malen und die Hingabe zur Gartenarchitektur mit für einen Laien ungewöhnlichen botanischen Kenntnissen.

In der Zeit des gesellschaftlichen Wandels kristallisierten sich Wertungen und Urteile zur Person Professor Grimms heraus, die ihm fehlinterpretierend den ständigen Kampf mit den Unzulänglichkeiten auf zahlreichen Ebenen des beruflichen Lebens als Kontakte zum System anlasteten. Bei hintergründiger Betrachtung ließen sich diese Verdikte schwerlich nachvollziehen und konnten letztendlich juristisch auch entkräftet werden. Gerade seiner Gradlinigkeit und dem kompromißlosen Einsatz für seine Klinik und seine Mitarbeiter war es zu verdanken, daß an der Klinik in Halle stets ein Klima der Kollegialität und uneingeschränkten Meınungsfreiheit herrschte, das die nahezu freie Entfaltung der kreativen Fähigkeiten der Mitarbeiter ermöglichte. Dennoch bedeuteten die Vorgänge für einen Mann, der in schwierigen Phasen seines Lebens und zur Aufrechterhaltung des klinischen Ablaufs für seine Mitarbeiter und an- 
vertrauten Patienten stets Verantwortung ohne Aufforderung, Pflichten ohne Rechte und Leistungen ohne Anspruch auf Anerkennung übernommen hatte, eine unüberwindliche Kränkung und verursachten Verbitterung.

Professor Grimm hat sich in seinem gesamten Arbeitsleben aufopferungsvoll seinen Patienten gewidmet. Er tat dies nicht nur als Operateur, sondern als Arzt des Vertrauens. Täglich bemühte er sich, vielfach unter Verzicht auf die verdienten Arbeitspausen und unter Einschränkung der Freizeit, in personlichen und einfühlsamen Gesprächen den Schwerkranken das zu bieten, was der modernen Medizin als Dienstleistungsunternehmen weitgehend verloren gegangen ist: menschliche Nähe, Hoffnung und Zuversicht. Seine Zuwendung zum Patienten war mit den juristisch absichernden modernen Aufklärungsgesprächen nicht einmal im Ansatz vergleichbar.

In der Geschichte der deutschen Mund-, Kiefer- und Gesichtschirurgie wird ihm als herausragendem Hochschullehrer und begnadetem Chirurgen der ihm zustehende Platz eingeräumt bleiben. Wir erinnern uns in ehrendem Gedenken an eine Persönlichkeit, die von Geradlinigkeit und intellektueller Redlichkeit geprägt war.

Gerhard Grimm wollte nichts anderes, als vor sich selbst $\mathrm{zu}$ bestehen.

\section{W. Müller, Erfurt \\ J. Schubert, Halle/Saale}

\section{Hornstein OP (Hrsg): Erkrankungen des Mundes. Ein interdisziplinäres Hand- buch und Atlas. Stuttgart: Kohlhammer (1996). 758 S., (ISBN 3-17-012995-3), DM 398,-}

En interdisziplinares Handbuch uber die Erkrankungen des Mundes, das eine fachubergre1fende aktuelle Synopsis vermittelt und so genauere Einblicke in das spezıelle Fachwissen der angrenzenden medizinischen Diszıplinen gewährt. lag bısher nicht vor.

Im Vorwort des Herauigebers wird deshalb zu Recht darauf hongewiesen, daß der Mund vom Eingangsbereich bis zum Rachen einen Teil der Gesichts- und Halsregion bildet, in der verschiedene anatomisch eng benachbarte Organsysteme multifunktionell zusammenwurken So bundeln sich im Mund als zentralem Bereich vielfaltige und komplizierte Funktionen zu elnem integrierten System, dessen potentielle Störanfalligkeit meıst erst be! den oft schmerzhatten Erkrankungen bewußt wird. O. P. Hornstein hat somıt aufgrund seiner uber 40 jahrigen klinischen und histopathologischen Erfahrung mit den Erkrankungen der Mundschlemmhaut die konsillarische Brucke zwischen den an der Oralmedizin direkt betellıgten Fachgebieten geschlagen und damit versucht. dem jeweilıgen arztlıchen Handeln ein interdisziplınäres Verständnis zu erschlıeßen.

Dieses Handbuch ist daher eher als en .,Manuale" fur den mit Patıenten befaßten Arzt, der sich rasch und lehrreich. aber nicht oberflachlich uber oralmedizinische Krankhettsbilder informieren will. aufzufassen. Es sst deshalb selbstverstandlich, daß alch auf 758 Setten das Spezialwıssen det verschiedenen Diszıplınen nıcht vollstandtg ersetzt werden konnte, so wurde $z$. B. bewubt unt histologrsche Abbildungen verzichtet.

Der Schwerpunkt des Buchs liegt auf der ätiopathogenet1sch-klınısch-diagnostisch und therapeutıch geglederten Darstellung der Krankheiten und Lastonen des Munds. In 43 Kapiteln werden folgende 10 oralmedizintsche Themenkreise abgehundelt. Einfuihrung in die Entstehung, Morphologle und Physiologe der Mundhohle enschliefblich Grundlagen der Diagnostik, angeborene orognathofiziale Mißbıldungen und Anomalien. Erkrankungen der Lippenreg1on. Infektıonskrankheiten der Mundschleimhaut, nicht-ntektuose Entzuindungen der Mundschleimhaut. Mundschleimhaut als Spregel innerer Erkrankungen. Hyperplasle, Prakanzerosen und Neoplasien der Mundschleımhaut, Anomalien und Erkrankungen der Zahne und Kiefer. Erkrankungen der Speicheldrusen und des lymphatischen Ruchenrings sowie neuro- und psychogene Affektionen in Lippen- und Mundbereich. In zahlreichen Tabellen und insbesondere hervortagenden klinischen Farbabbildungen werden die diagnostischen Unterschiede der verschiedenen Lasionen. Anomalıen und Krankheltszeichen zurreftend erfallit. Der Darstellung der Theraple sowle der psychosomatischen Probleme, dic an Bedeutung immer mehr zunehmen, wird viel Platz eingeraumt. Jedes Kapitel enthailt ein weiterfuhrendes Literaturverzeichnis. das jedoch nicht immer die neuesten grundlegenden Publikatıonen beruchsichtıgt.

Da der Rezensent unter dem Zwang zur Beschränkung bes der Abfassung einer Buchbesprechung unmoglich alle 43 Kapitel im einzelnen wurdigen kann, seien somt lediglıch Anregungen zu wunschenswerten Uberarbeltungen erlaubt.

Im kurzen Kapitel uber die bildgebenden Untersuchungen im Kiefer- und Schadelbereıch vermißt man die Darstellung der immer noch wichtigen, durch keine anderen Verfahren zu ersetzenden Kontrastmitteldarstellungen wle Anglographie und Arthrographı. Beı den nicht-10nısierenden bıldgebenden Untersuchungen wird die Sonographie im B-Scan-Verfahren, gemesven an shrer hohen klınischen Bedeutung. viel zu knapp dargestellt. Gerade mit den heuttgen Moglichkeıten des 13-MHz-Schallkopfs konnen die Weichteilstrukturen des Munds und Halsbereichs nicht nur 2-, sondern inzwischen auch 3-dimensional aufgezeichnet werden. Hier wären entsprechende zusatzliche Abbildungen z.Z. ist keine sonographische Abbildung vorhanden - für dieses Buch als ,.Manual" unbedingt wunschenswert.

Im Kapitel uber die angeborenen orognathofazialen MiBbildungen und Anomalien werden von dem interdıszıplınären Autorenteam, $A$. Eckstein, H. D. Mußig und O. P. Hornstem, insbesondere die Lippenkiefergaumenspalten anschaulich und auf das wesentliche konzentriert vorgestellt. Teilfragen, wie die langfristige Beurteilung der unterschiedlıchen Operatıonszeitpunkte innerhalb des Gesamtkonzepts der Rehabilıtation von Patıenten mit Lippenkıefergaumenspalten. werden nur marginal behandelt. Etwas glücklicher ware es, im Sprachgebrauch nicht mehr von ",Mißbildungen“, sondern besser von ..Fehlbildungen" als dysontogenetisch bedingten Verảnderungen der Normalstruktur zu sprechen.

Im Kapitel über die Neoplasien der Lippenregion werden die Basaliome als Tumoren mit enner .,ergentümlıchen Mittelstellung" zwischen benıgnen und malignen Epithehomen der Haut beschrieben. Insbesondere wird auf das Fehlen von Metastaslerung und tumorbedingter Kachexie hingewiesen. Diese Darstellung des Basalooms und die damit verbundene gewisse Verharmlosung entspricht nicht der tatsachlichen Dignitat dieses haufigsten malignen Tumors der Haut. Obwohl ene Metastasierung der Basaliome aukerst selten ist, sind bisher doch uber 170 Falle eines metastasierenden Basalıoms beschrieben worden. Bevorzugte Metastasierungsorte waren dabeı Lunge. Knochen und Lymphknoten. Aber duch in Leber. Milz. Nebenniere sowle Pankreas. Schilddruse. Nieren und in anderen Organen wurden Metastasen nachgewiesen.

Sehr anschaulich wird in dem Kapitel uber die viralen Infektionskrankhetten die HIV-Infektion mit ihrer Epıdemiologle. Atiopathoge- 